Fuzzy Sets and Systems 2 (1979) 37-52

(C) North-Holiand Publishing Company

\title{
ENHANCEMENT OF COPING THROUGH BLURRING ${ }^{1}$
}

\author{
Manfred KOCHEN \\ The University of Michigan, Ann Arbor, MI 48109, U.S.A.
}

Received July 1978

Revised August 1978

\begin{abstract}
When problem situations are imprecise, peopla use mental processes closer to coping than to exact, logical-deductive reasoning. We sketch new wavs of explicating the process of coping. This leads to a broader definition of complexity than is generally used in computer science. We present as the essence of coping a normative model for shifting and changing focus of attention. To cope is to transform a complex situation into blurred representations that are simpler and less worrisome. The process of "thinking cf" such shifts in problem-representation is also improved when the level of specificity and precision in the focus of attention varies.
\end{abstract}

Key Words: Inexact reasoning, Coping, Complexity, Attention, Representations, Precision, Blurring.

\section{Introduction}

As a person steers the course of his life from day to day, he spends more time "coping" than he does "reasoning" or "problem-solving". Yet, it is the attempt to understand reasoning which has most strongly motivated both descriptive and normative modelers. The long history of forma! logic has had its greatest successes when it did not attempt to describe or priscribe for human reasoning, much less coping behavior. The crowning achievements of modern logic lie in delineating its own logical limits. Steps toward a mathematical theory of evidence have been less spectacular since Leibniz's hope, three centuries ago, that it could be developed to the point where it helps lawyers. Yet, formal logic comes closer than any other mathematical structure to a normative model of reasoning. If its success in that endeavor has been less than perfect, that may be the result of its inapplicability to the "imprecise situations of real life"[7].

There have been many attempts to create normative models of how to reason or think rationally when we can only rave approximate, partial or blurred knowledge of reality. The importance of the distinction between intuition and

\footnotetext{
${ }^{1}$ A shortened and substantially different version of this article was presented at the IEEE Symposium on Fuzzy Sets and Applications in. New Orleans on December 8, 1977, chaired by M. Gupta. It was preprinted in the Proceedings under the title "Imprecision in coping and attending processes". Sections that cuplicate the earlier version are reproduced here with permission of the publisher.
} 
rigorous analytic thinking and the need for both has been recognized for a long time $[12,24,25]$. Intuition enables us to ask questions or to make bold assertions, while we prove our assertions in a more precise and algorithmic way, and research is the result of both processes [19]. Another approach has drawn a similar distinction between horizontal and vertical thinking [2]. Yet another approach that has led to impressive advances was based on the idea of a mixed strategy of making decisions in choosing by lottery [4]. The approach which has attained the greatest popularity, however, is Fuzzy Set Theory, the literature of which has grown to over 800 articles [8] since the first seminal paper appeared [28].

What is imprecise about the situations of real life is how they are perceived, interpreted and evaluated. The situation of the fleeing patron of a burning, crowded theater could be objectively measured by others with great precision. To him, the situation is vaguely threatening: he may not know exactly where the fire is or how serious it is; he may not know exactly how to get to the nearest exit, or the effect of his fleeing behavior upon all the others, or theirs upon him. He is unlikely to resort to reasoning in this situation, partly because he is not certain that reasoning will help him survive. (This is not to say that he ought not to stay cool and resort to reasoning.) Yet many people can cope with such situations, and not necessarily only those with superior reasoning or precise thinking abilities.

The more complex the situation, the more difficult it is to perceive with precision. Ainong the most complex social situations are those arising in the management of organizations or where several people with diverse world images, values, action styles are all critically involved. An example is how two or more rescuers can best help the victims of a disaster such as the burning theater.

The study of behavior in such complex situations, both descriptive and normative, should be central in the behavioral, social and information sciences (BSIS). Yet the more mathematical researchers in those sciences seem not yet to have found a promising line of investigation. One reason for this may be their striving for precision and their abhorrence of vagueness. Management scientists often model decision-making by a series of stages. These include problem-formulation, statement of objectives, fact-gathering and analysis, determination of feasible alternatives (optional courses of action), evaluation of alternatives, etc. Practicing managers often adopt as their ideal "management by objectives". The essence of this is goal setting. Vague and poor goal definition is widely regarded as a common flaw. It is to be avoided at all costs. Yet, too much precision and (apparent) clarity may be as ineffective as too much vagueness and confusion. The attempt to perceive a complex situation with an "inappropriately high" degree of precision and clarity may fail, with costly consequences.

To clarify what is meant by an appropriate degree of precision in attending to and coping with complex situations we first try to show that to cope is to formulate or reformulate problem-statements corresponding to a worrisome situation in order to reduce its complexity. We then focus on one important kind of problem transformation, "blurring", and show how that can bë explicated with 
the help of concepts from Fuzzy Set Theory. We then investigate the process by which such problem-transformations cre thought of and try to show that this requires an alternation of concentration on precisely specified aspects of a situation and relaxation that affords a more blurred and suid image. This central attending process is conceptualized with the help of ideas from Fuzzy Set Theory. In doing this, we hope to illustrate the potential utility of Fuzzy Set Theory (FST) for BSIS's more -generally. We hope to suggest also that such "applications" can enrich the further development of FST.

\section{What does it take to cope?}

The term "coping" is widely used in ordinary discourse. But the concept has not, to our knowledge, been studied scientifically. We hope here to add some clarity to our understanding of what it means by showing that to cope is to formulate or reformulate "problem-statements" corresponding to a worrisome complex situation to reduce its complexity and worrisomeness.

A recent article entitled: "Mathematics and computer science: Coping with finiteness" [16] comes closest to defining the term by its use in the sense intended here. Though the concept is essential for mental health professionals, the term "coping" does not appear in the subject indexes of major books. Mention of it or research related to it is strikingly absent from the work supported by NIMH since 1967 [26]. In an important study of how people say they handle worrisome and unhappy situc.tions [11], their responses to questions were categorized into three groups: passive reactions (e.g., denial or taking one's mind off the situation, doing nothing, keeping on worrying); coping reactions (thinking things through, attempts to alleviate, turning to formal or informal sources for help); prayer (e.g., mobilizing internal resources, resignation). Thirty to forty percent of the respondents said they "coped", most of them by informal help-seeking, most commoniy family and friends. Direct coping was found to be more common in men, younger people, the more educated and in those with higher incomes.

To cope with a worrisome situation is to attend to it and to mobilize and organize the resources necessary to reduce its worrisone aspects. If the first step were to expend time and effort to analyze, clarify, reduce what is worriscme about the situation into aspects that are precise, familiar and easily handled, as a scientific technician tries, ideally, to solve a routine scientific problem, then the situation could become more worrisome. It is not merely a question of the coper's limitations in clarifying the nature of the task or problem facing him, nor the delay incurred by trying, but the basic limitation of how precisely the "worrisomeness" of a complex situation can be perceived that bounds the use of an unimaginative, systematic approach.

Consider the classi zal example of the tennis tournament problem: if there are $n$ tennis players in a tcurnament, how many contests must be played between two players, assurning nc ties, with winners of contests playing one another uritil a 
final winner emerges. A straightforward analysis of the problem, precisely as stated, is to say that in the first round there are $\frac{1}{2} n$ contests, in the second round $\frac{1}{4} n$, etc., for a total of $\frac{1}{2} n+\frac{1}{4} n+\frac{1}{2} n+\cdots$ with examination of cases when $n, \frac{1}{2} n$, etc., are not even. The problem-statement can, however, be transformed by noting that every player but the final winner-a single person-must lose in exactly one contest. Hence the number of contests is the number of losers, or $n-1$. There is something less precise about asking for the number of losers at the end than asking for how many winners there are left at the ends of each round; it means thinking about the completed tournament in retrospect rather than describing how it progresses. What is even less precise is the mental process of "hitting" upon the idea of asking for a number of losers at the end.

This example is often used to show that scientific creativity is far more than following systematic analytic procedures. The kind of problem-transformation used in this example might be called "passing to the negative". Another useful example of a problem-transformation, which involves both "passing to the negative" and "blurring" was used to show the advantage of lateral (intuitive) over vertical (logical-deductive) thinking [2]. Here a girl is faced with the following worrisome situation. Her father's creditor wants to marry her against her will. She has agreed to participate in a lottery to decide her fate. The creditor meets her on a pebble-strewn path. He is to pick up a black and a white pebble and place them in his pouch. She is to select one of the two from the pouch. If she chooses black, she is to marry him, and her father's debt is absolved. If she chooses white, she need not marry him, and her father's debt is also absolved. If she refuses to play, her father will be jailed. Sharp-eyed with fright, she catches the creditor picking two black pebbles and placing them in his pouch. How is she to cope?

Ordinary, systematic analysis allows for no ingenious ways out. The problem may be "blurred" as follows. She picks a pebble but does not look at it. Instead she artfully drops it so that it gets mixed with the other pebbles on the path. Now she passes to the negative by saying: "How clumsy of me. But no matter. We can see what I dropped by looking at the pebble remaining in your pouch. It must be the opposite of what I dropped".

The notion of "blurring", possibly one that is only superficially analegous to the above, has been scientifically studied in the context of vision [13]. It was found that a coarsely sampled and quantitized portrait could be recognized more easily after it is squinted at, defocussed, viewed at a distance. This has, of course, been practiced by painters for a long time. What appears to be happening is that noise bands which are spectrally adjacent to the picture's spectrum suppress recognition. Thus, by dividing a photograph of Lincoln into squares and replacing each square by the average light intensity or density of that region in the original the resulting picture becomes very hard to recognize. This can be improved by removing noise near the spectral frequency of the picture (filtering past its Fourier transform and transforming back). 
The improvement in recognition due to blurring is due to visual integration in the nervous system. In audition, people do worse in detecting a pure sine wave of any frequency when there is a second sine wave of a frequency within an octave of the first one [29], and removing such "noise" signals enhances detectability. Blurring, which removes the noisy signal, appears to help most when such noise is present. If a subject is presented with two figures that are sufficiently similar so that he has difficulty in discriminating them, say (and), then adjoining a noise signal that resembles one of them makes the discrimination task much easier, e.g., ((vs)). But it resumes its former difficulty as the original and the noisy signal are separated.

There may be fruitful analogies to situations such as the lottery involving the girl and her father's creditor; here a "noisy" signal in the form of the creditor picking up two black pebbles, as seen by the girl, is in the "spectral vicinity" of the main "signal", the color of the pebble chosen by the girl. To drop the chosen pebble, to be mixed with many others, is to remove the noise signal, to destroy unwanted information, which is what occurs in blurring.

The analogy should not be stretched too far or perhaps taken too seriously. Cognitive blurring or intellectiai ciefocussing may differ from perceptual blurring, of a forest-literally as in the case of a photograph or mosaic and figuratively as in the case of a complex puzzle facing a detective, scientist or doctor-only if we blur the trees, fuse the details so that they become subordinate to and coordinated into the whole. Even in the most complex of competitive board games, such as GO, experts know precisely what move they are going to make before they make it. By contrast, even in the simplest of zero-sum games, such as the one characterized by $\left(\begin{array}{cc}8 & -12 \\ -11 & -9\end{array}\right)$ as the payoff matrix for the player choosing one of the two rows (against a player choosing one of the two columns) the row-chooser does best, not by knowing precisely which row to pick and doing it, but by spinning a spinner on a disk divided into two sectors, one of which occupies $\frac{1}{11}$ of the disc; if the spinner lands by chance in the smaller sector, he chooses the first row, otherwise the second. Neither he nor his opponent can know beforehand what he will choose.

The spinner-disk device could be designed according to degrees of membership over the various sectors or according to a possibility distrikution. In this way, we can perhaps not only overcome the limitations of imprecision but use imprecision to advantage, for enhancement. Neurophysiologists have begun io ask if this is not a principle according to which nervous systems might be organized. Social scientists who are skeptical of attempts to define corcepts such as "power" or "consensus" with logical precision may find in the expressions of humanists (e.g., artists, novelists) more persuasive ways of capturing their basic concepts.

The essence of how we cope is probably in how we attend to a situation. To attend to a situation means first to perceive, interpret and evaluate it. To reduce its worrisomeness is to focus attention on those aspecss of the situation that account for most of the worrisomeness. The more complex the situation, the more 
imprecisely we tend to perceive, interpret and evaluate it, and the more difficult it is to focus attention appropriately. We are sometimes helped by deliberate lapses in attention focussing or by attention defocussing. When attention is focussed, the key question concerns the mechanisms controlling on what it is focussed, and these control mechanisms need not always be precise.

\section{What makes a situation complex?}

At least six difficulties make a situation complex: (1) the difficulty of specifying the set of alternatives from which to choose a possible "solution" or coping actions; (2) the difficulty of specifying the set of properties a "solution" should have; (3) specifying, finding and using the knowledge that is necessary and sufficient-or at least helpful-in finding a "solution"; (4) bearing in mind the various deadlines-maintaining awareness about what to attend to at all times and when to do what; (5 and 6) realizing potentialities and constraints of information processing capacities and of psychosocial interactions. We show in this section that each of these difficulties is in the nature of making our perception and evaluation of the situation less precise, or that they call for shifts in point of view, and that efforts to make such shifts are hampered by excessive exactness.

\section{1}

To produce a set of alternatives or possible "solutions" for consideration requires creative ingenuity. In complex situations, that involve several people, it also reguires the ability to resolve conflicts among diverse world-images and to shift viewpoint during the agenda-setting process. An example of a non-complex situation is that faced by a student trying to solve a well formulated problem at the end of a chapter in a textbook. The problem may be to determine if 541 is a prime number. The set of possible solutions is (Yes, it is a prime; No, it is not). The real problem facing the girl in the lottery involving her father and prospective marriage is more complex because it does not exclude the crafty solution she eventually resorted to.

The example of how two or more rescuers should cope with a disaster involving many victims may clarify the need to resolve conflicts in even more complex situations. Prior to deciding on a course of action, the group of rescleers may create an agenda; this may be explicit, as set by a leader who convenes them to organize their joint effort, or implicit when each rescuer pursues his own path in a kind of silent tzam work among people who work together well, or in a buzzing confusion among people who do not. A proposed set of options for the group to choose from depends on the proposer's point of view. One orientation, $A$, would lead one rescuer to propose the following alternatives for the group to choose from: $A=\{A 1:$ Tend first to those who bleed most profusely; $A 2$ : Tend first to 
those in greatest agony or pain; etc.\}. A second rescuer with another orientation, $B$, might propose $B=\{B 1$ : Tend first to those who, if they survive, are most likely to join the rescuers and help others; $B 2$ : Tend first to those likely to contribute most to society, etc.\}. The proposer of $A$ may not have thought of $B$ as a different way of perceiving the situation. He might be willing tc shift his viewpoint. If not, he might have to compromise with $A$ or cope with potential conflict some other way.

In any case, neither the point of view, nor the proposed set of options that reflect it, nor the alternatives within a set of options are completely precise and unambiguous. If they were, they might hinder rather than help in both the $p$ ocess of reaching a group decision and in carrying it out by ruling out better solutions, such as the one adopted by the girl in the tv'o pebble lottery. To articulate the point of view implicit in $B$ as one that favors first aid to those of "greatest potential social utility" may engender more controversy, delay and result in greater overall loss of life than leaving it vague. To specify exactly what rate of blood loss, an excess of which justifies top priority attending to, may be impossible or counterproductive to apply in practice.

\section{2}

To specify the set of properties a "solution" should have, when more than one action is involved, may require the resolution of value conflicts. In the simple situation of deciding whether 541 is a prime, the property of the solution, namely the truth that 541 is a prime, is an explicit part of the problem statement. That is part of what makes it well-defined and simple. In more complex situations, the desiderata of an acceptable course of action are not always clear and if several actions are involved, they may be controversial.

One rescuer may value a human life regardless of the goodness or quality of that life; if he had to choose between a victim bleeding profusely who is a known criminal or public enemy and another victim in great pain who is also dearly loved, he should tend to the one in greatest need first. Another rescuer may place greater value on lives of younger more creative people than on those of very old and dependent people. These values would influence a rescuer's preferences among a given set of alternatives. If two rescuers are to abide with a solution, or set of rules governing behavior, that is to apply to all rescuers, then they must somehow adjust these differences among value orientations. Differences in values are, however, seldom as sharp as partisans make them out. The main contribution to complexity lies in the imprecision of the perceptions and evaluations of the criteria for a good solution.

To specify, find, and use knowledge that is necessary and sufficient to find a "solution" rarely involves precision. We seldom know exactly what we need to 
know until we are nearly face-to face with it. The knowledge itself is seldom very precise either. Only in simple problems, such as deciding if 541 is prime, is the knowledge or method needed implicitly specified in the problem-in this case by the location of the problem at the end of a chapter that aimed to teach the method. Prescribing the dosage of an intravenous solution of morphine to administer to a victim does not warrant a great deal of precision, for dosageresponse relations, where known, are at best crude approximations. The complexity of the interacting biochemical systems on a person is such that an optimal dosage cannot, perhaps even in principle, be calculated with exactitude.

\section{4}

Timing of required actions is perhaps the greatest source of difficulty in a complex situation. We may have resolved to wear seat belts in a car or to refrain from smoking, but unless that resolution becomes salient and compelling at the precise moment we start to drive a car or experience the urge for a cigarette, it is of no value. If we don't think of starting a course of action in time to finish by a known deadline, we need not start it at all. The process of attending to something is critical to successful coping. It is far more difficult to attend to very precisely specified actions or states, especially if there is a great variety of these, than to attend to more vaguely specified states and actions, provided they are not too vague. The same applies to specifying the time when certain actions should be taken. Taking a prescribed medication at exactly $8: 18 \mathrm{a} . \mathrm{m}$. on a certain day is harder to attend to than taking it in early morning of that day.

\section{5}

Psychosocial and informational limitations as well as potentialities are realized only vaguely. Stress generated by agonizingly difficult choices may interfere with a decision-maker's ability to properly evaluate alternatives [14]. If the decisionmaker is aware that risks of a situation are serious but has no hope of finding a solution, he will be under high stress and cope via "defensive avoidance"; if he has hope of finding a solution but doesn't have enough time, he will be under moderate stress and cope vigilantly, thoroughly searching, appraising and exploring all the alternatives. The best coping strategy is vigilant attention, with open-mindedness to both supporting and opposing information. People will do this if they believe that serious risks are involved, that a solution or good way out can be found, and that there is enough time. Stress as the mediating factor is an inherently imprecise quality. It is a subjective feeling, even though physiological correlates such as heartrate, can be precisely measured. Similarly, awareness of one's information-processing limits is imprecise. Few of us can specify precisely and in general how much information input will overload our input or memory capacities.

To cope with complex situations, then, is to perceive, recognize, evaluate it with 
less than $100 \%$ precision and to mobilize, organize and bring to bear resources in ways that should not always be specified with great precision either. The most important aspect of coping is the ability to mobilize, ${ }^{2}$ focus/defocus and shift attention to aspects of a situation that were previously only dimly or vaguely perceived. This process of shifting attention is executed by an executive controller at higher levels of the nervous system, and is in itself, subject to shifts in precision of attention focus.

\section{Attending processes}

When we observe a person shifting his attention, concentrating, going into a state of reverie, we regard his behavior to be governed by an attending process. To specify an attending process, we need to specify as functions of time: (1) what is being attended to: (2) the output of attending; (3) how what is being attended to is "kept in mind" and (4) the instantaneous intensity of concentration.

\section{1}

What is attended to can be described at several levels. Let us arbitrarily select an ontological level at which the attender's state is specified by $n$ variables, $s_{1}, \ldots, s_{n}$, such as the brightness of a dot, and its location, the frequency of a sound and its intensity, etc. At a different level we might choose the number of victims in a disaster, the rate of each victim's blood loss, the number of rescuers, etc. With each state-variable is associated a secondary variable, relating it to the attender's perception of it. Thus, to $s_{1}$ are associated $\mathrm{sal}_{A}\left(s_{1}, t\right)$ or the saliency of $s_{1}$ to attender $A$ at time $t$, and $\operatorname{aff}_{A}\left(s_{1}, t\right)$, the affect of $s_{1}$ for $A$ at $t[15]$. A attends to those state-variables at time $t$ which, for him, have high saliency and high affect. What is attended to is an aspect of the situation, specified by the name (address) of a state-variable.

To say that what is attended to is a state-variable, is to say that its values are monitored or tracked for a time. But what does it mean to monitor or keep track of a variable? Suppose that $s_{1}$ is the frequency of an acoustic signal. One level above that, there corresponds to $s_{1}$ another physical variable, such as the intensity $s_{1}{ }^{\prime}$ of vibration at a particular point on the basilar membrane of $A$ 's auditory system. That may, in turn, be made to correspond to another physical variable $s_{1}$ " relating to coneentrations of certain neurotransmitters and electrical potentials at

${ }^{2}$ In another study [17] 10 subjects were each asked 5 questions the ansv'er to which they could provide only by looking them up in books through which they were able to browse, and in which they had seen the answers prior to being asked the qrestions. Of the 50 problem-situations, only 28 were coped with by producing the right answer. Of the 22 remaining over 15 were the result of not thinking of asking for the book that contained the answer. 
specific points of $A$ 's nervous system. For $A$ to attend to $s_{1}$ is for $A$ 's nervous system to connect or couple channels at those points where $s_{1}$ " can be observed with channels associated with a higher executive controller in the nervous system. Possibly, it is $s_{1}$ " that is monitored or tracked, and though the correspondence between $s_{1}$ " and $s_{1}$ may not be $1-1$, it is as if $s_{1}$ were monitored.

\section{2}

To attend to $s_{1}$, then, is to specify what channels should be connected to the channels of an executive controller. The output of an attending process is two fold. First, it is a signal indicating that the connection was made. Secondly, it is the value of a signal generated by a chain of internal stimuli starting from $s_{1}$ through that connection. It is the end result of the chain of signals "flowing through" the pathways thus created. The channels to be coupled may be recognized by their chemical signatures or their anatomical locations.

\section{3}

But how does the executive controller specify what to attend to? In line with modern psychological thinking we regard this as an active process, one initiated by $A$ rather than one in which $A$ responds or sacts to some property of $s_{1}$. It is $A$ who assigns a saliency and affect to each state-variable. $A$ filters, at fairly low levels, the state-variables of their images and passes to an executive controller only those of high saliency and affect. Because the number of state-variables can be very large and their combinations even larger, there is likely to be considerable aggregation, even at lower levels. Thus, instead of frequency, light flux, etc., what is attended to is rate of blood flow, severity of pain. In addition to being aggregated, the state-variables are also "blurred", as if they were squinted at in order to obtain a detail-suppressing overall impression, as discussed in Section 2. Thus, "bleeding profusely" becomes a general impression that is "kept in mind" by an executive controller, which connects its channels not with one but with several other channels in a way that corresponds to a "fuzzy intention", enabling $A$ to attend to victims who are bleeding more or less profusely. (The hedge "more or less" has been analyzed with the help of Fuzzy Set Theory.)

The concept of intention is central. An intention is a statement, to which $A$ is committed, about the properties of an adopted program that refers to that part of the program that has not been executed [18, 20,21]. An intention as well as a program can be stated with a degree of care that varied from perfect specificity, precision, completeness, accuracy (consistency) to great generality, imprecision, incompleteness and inaccuracy or inconsistency. A non-fuzzy intention might be to monitor the blood loss rate of a particular victim while a fuzzy intention might be to survey the entire scene following the catastrophe to spot victims who seem to be most in need to help. Similarly, the value of a variable can be monitored, 
kept track of with different degrees of precision and reliability. Thus, the tleeding rate of a particular victim could be carefully watched with considerable precision at all times or simply glanced at occasionally to check if it is still profuse or slowing down.

\section{4}

When $A$ concentrates on some aspect of his worrisome situation, he forms a precise intention that specifies carefully what is being attended to and he monitors these specified variables very carefully. The total energy and information processing capacity that is available during $\ddot{a}$ given time interval is fixed. Maintaining a connection to keep track of each variable and tracking with a given degree of precision takes a certain amount of these fixed resources. This amount increases with the degree of care or precision. Hence, the number of variables and the degree of precision are subject to trades. This corresponds to such laws as the Bunsen-Roscoe law: the intensity of a stimulus times its duration given rise to a constant number of dots that subjects respond to correctly $50 \%$ of the time, up to a critical point [10]. We can concentrate very intensely on a narrow aspect of the situation for a limited period of time and this may lead to great feats in which the mind functions as an algorithmic processor, proving conjectures. But this narrowness may blind the mind to other, totally different, approaches or perceptions of the situation. We can also relax, and switch from intense concentration to very playful fuzzy attending. In those periods, some minds may leap to great intuitive insights to create bold, often fuzzy, assertions, to ask profound questions.

The attending process may play a major role in how we cope with complex situations. How we attend to those aspects of a situation we find worrisome, how we select a set of alt ${ }^{-n}$ native courses of actions, etc.-above all how we switch back and forth between attendance with sharp concentration and fuzzy relaxation-may determine how effectively and efficiently we reduce the worrisomeness of a situation.

\section{Reduction of complexity}

When a worrisome situation is not terribly complex, it is not difficult to perceive it with the kind of precision required for successful use of reason or vertical logical thinking [2]. As we have tried to show in the preceding section, the more complex a situation, the more fuzzy its perception, interpretation and evaluation. Consequently, coping is more likely than precise reasoning to help reduce the worrisomeness, because coping involves perceptions and responses with a more appropriate degree of fuzziness or imprecision. We should like in this section to present some arguments for this proposition. 
Perhaps the simplest idealized example of a minimally complex situation is that of determining whether a given large number $n$ is or is not a prime. This could be transformed into a worrisome situation by suitably challenging and motivating a group of mathematicians, giving them appropriate deadlines, amplifiers to their information-processing abilities (e.g., computers, programmers, literature searchers, assistants, etc.). The problem is decidable and known to be computationally very complex. For example, to test if $n=2^{128}+1$ has a factor (useful for designing certain codes in space communication) by testing one after the other up to $\sqrt{2^{128}+1}$ would take about 2000 years on a high-speed computer [16]. With the help of sophisticated mathematical techniques, the smaller prime factor was found to be $59,649,589,127,497,217$ after about $1 \frac{1}{2}$ hours of computer time $[16,22]$. This computation time still increases exponentially with $n$. It is an inherently complex problem to find a prime factor.

The notion of comple :ity we introduced in Section 2, however, goes considerably beyond the way comp exity is defined by computer scientists. The problem of whether $n$ is a prime is in that sense complex only because of property [4], bearing in mind that there are deadlines and [5], that information processing capacities are constrained. It is simple in that (1) the set of possible solutions, (2) the properties a solution should have and (3) the knowledge necessary to find some solution are precisely specified. The computational complexity is, however, sufficiently great to affect these aspects.

To cope with this complex situation, the mathematicians may decide to transform the problem. They may change the original set of possible solutions from (No, Yes) to (No, Possibly, Probably, Very probably, Almost certainly, etc.), by the following method. They "guess" a number between 2 and $\sqrt{n}$ and test if it divides $n$. If it does, they are certain that $n$ is not a prime. If it does not, they hypothesize, or assert with uncertainty, that $n$ is a prime. If they find this uncertainty still too worrisome, they guess a second, different number between 2 and $\sqrt{n}$ and repeat the test. Finding that neither number divides $n$, they are more certain. If the residual certainty is still too worrisome they proceed to a third trial, until the residual worrisomeness of the situation has been reduced to a level they can accept.

There is an image of mathematics as a field of study that is so exact that only a zero level of residual uncertainty will do. That this is unrealistic is apparent from the complexity of modern proofs: when a proof is sufficiently long and involved, the probability that its author or those who checked it made no errors at any point is no longer zero. Even with the help of computers, which have already played an important role in major mathematical problems (e.g., settling the 4-color conjecture) the residual uncertainty should no longer be regarded as strictly zero. Thus, even in the most exact of the sciences, there are situations in which coping is more appropriate than traditional, precise reasoning.

Consider the following algorithm (see Fig. 1) for coping with the determination 


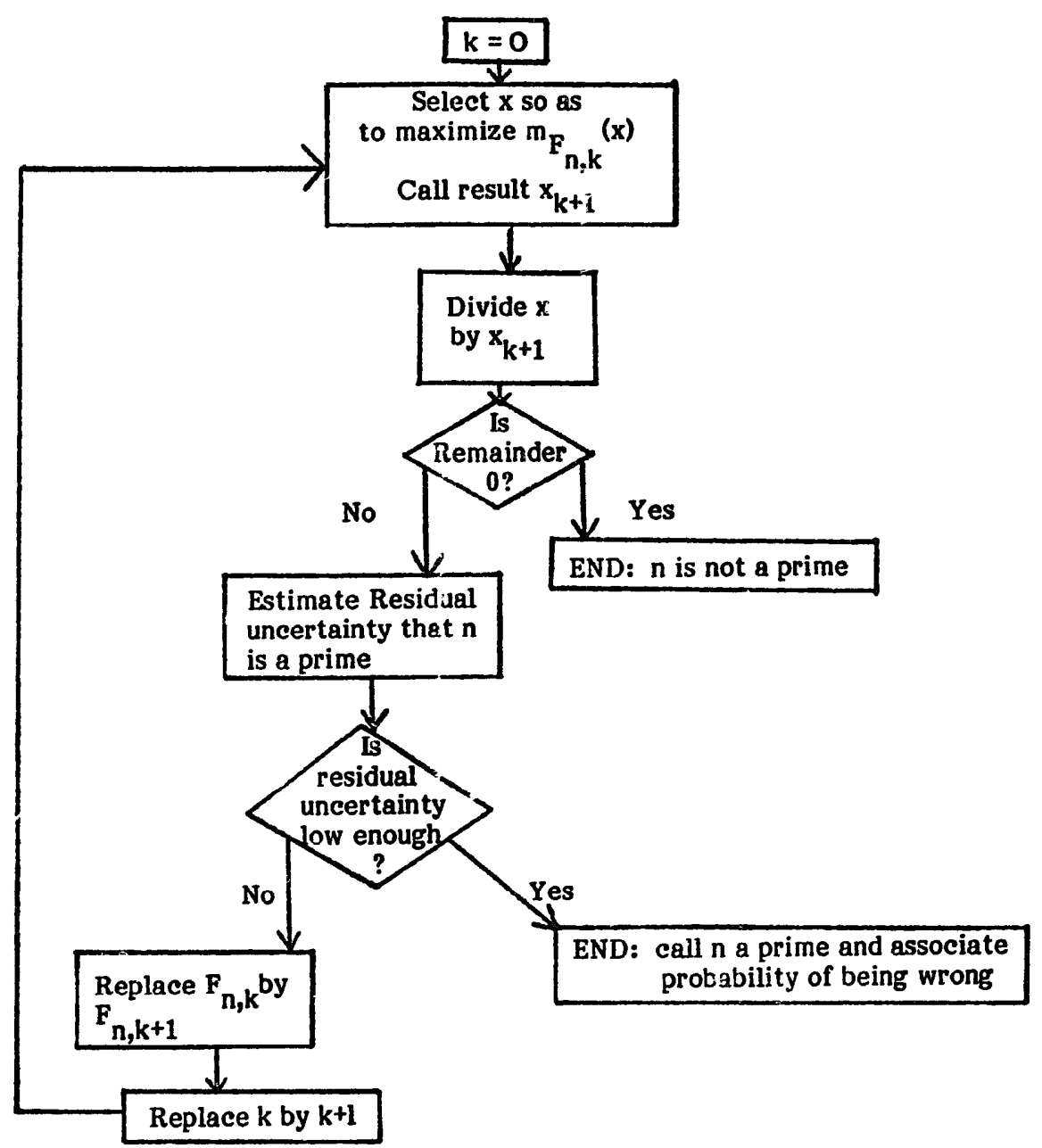

Fig. 1. An algorithm for "guessing" whether a number is prime.

of whether $n$ is prinie. Let $F_{n, k}$ be the fuzzy set of numbers, between 2 and $n$, other than $x_{1}, \ldots, x_{k}$, specified by the membership function:

$$
\begin{aligned}
& m_{F_{n, k}}(x)=0 \text { if } x=x_{1} \text { or } x_{2} \text { or } \cdots \text { or } x_{k}, x \leqslant 2, x>\sqrt{n} \\
& \text { the strength of belief that } x \text { divides } n .
\end{aligned}
$$

We may interpret $m(x)$ as specifying the weights or sector sizes of a spinner to be used for selecting a value of $x$ with a "probability" or degree of membership that is proportional to some mathematician's intuition that $x$ divides $n$. Call the outcome $x_{k+1}$. Fuzzy set theory gives little guidance about how to determine this function, though it helps to derive implications about it.

The complexity of this algorithm is clearly much less than the best that can be done by number theoretic techniques aimed at removing all the uncertainty. The computing time need increase no faster than $n \log n$. Thus, coping, which is essentially dealing with the less than perfect exactness of how we perceive, 
interpret, evaluate complex situations, can be effective in reducing certain aspects of complexity.

Of all the ways of transforming or reinterpreting a situation or problemformulation, how can a group of copers arrive efficiently at one that works? What can lead mathematicians to replace a time-honored striving for either (No, Yes) as possible answers to "Is $n$ prime"? by (No, Possibly, Probably, etc.)? What can lead the girl in the two-pebble lottery to replace (Pick a pebble and look at it, Refuse to pick) as the set of possible actions in her predicament by adding to these choices the action she took? Under what conditions does a group of rescuers act nearly spontaneously as a team to perform heroically in coping with the effects of a disaster while others bog down in chaos and strife? An answer probably lies in the manner in which the copers attend to the various aspects of the situation. Focussing attention too rigidly, too intensely and for too long on one representation of the problem-e.g., mathematicians locked into the (No, Yes) alternative searching for special properties of the number whose primeness is in questionmay be counter-productive.

\section{On the development of Fuzzy Set Theory: interactions with "applications"}

Fuzzy Set Theory (FST) is a mathematical theory or a tool rather than a scientific theory because it lacks an empirical/experimental foundation. It has been claimed that possibility theory may stand in the same relation to FST as probability theory stands to measure theory. Yet, anything corresponding to mathematical statistics has yet to be developed. The development of FST is likely to mature if it deals with some particular problems that present the theory with unique challenges. The problem of clarifying progress for attending to complex, worrisome situations is such a problem. Study of that problem is as likely to enrich FST and deepen our understanding of the problem. It is more appropriate to treat FSD as a means to an end, such as clarifying this important scientific problem, rather than an end in itself. A scientific understanding of attending processes is likely to require some understanding of how memory and control organizes itself in our central nervous system. General principles according to which "memory" organizes itself might arise from the use of formalisms using analogies to thermodynamic nets in physical chemistry $[5,9,23]$. If FST could help in the formulation of these principles, it would thus be greatly enriched. But general principles still allow for many possible alternative implementations and to find the alternative "chosen" by nature requires a detailed study at the level of neural nets and neurobiochemical pathways (e.g., [6]). We may, at best, find FST necessary but not sufficient in advancing our understanding of major scientific problems.

Attending processes can be studied experimentally at several levels. There are simple designs of psychological experiments to test whether people who use 
blurring or defocussing mechanisms can under certain conditions cope better than people who use precise algorithms. Attention-shifting can be studied at a physiological level by observing eye-movements and extending techniques used to probe short-term memory and tracking behavior. At a molecular level, one can search for specific molecules on the surfaces of neurons that enable them to make the selective functional contacts suggested by the coupling model described previously. Here a key question concerns the level of specificity of such molecules, a notion similar to fuzziness [1].

The straightforward "application" of FST to the pebble problem with the choices (Pick a pebble and look at it; Refuse to play) does not help the girl out of her double bind, in which no matter what she does she loses. FST has not yet matured to the point where it can helr to explain how the girl could invent the third option: "Pick a pebble but don't lcok at it and drop it". Once the third option is included, its superiority to the other two options is obvious, and FST is not needed to prove that.

Yet, models for coping and attending processes may suggest further exercises for sharpening the tools of FST, particularly the type of FST developed by Saaty [27]. An example of a problem in attention that illustrates the use of FST is furnished by how to divide one's attention between three reports- $A, B$, and $C$. The attender may judge $B$ to be half as salient as $A$, and $C$ just as important. The eigenvector of the reciprocal pairwise comparison matrix is $(0.25,0.50,0.25)$. The attender may view $B$ as five times as emotionally charged as $A$ and $C$ on a par with $A$; the eigenvector of that matrix is $(0.45,0.09,0.46)$. If asked to compare saliency and affect, he may give affect twice the weight, resulting in the eigenvertor $(2 / 3,1 / 3)$. Multiplying

$$
\left(\begin{array}{ll}
0.25 & 0.45 \\
0.50 & 0.09 \\
0.25 & 0.46
\end{array}\right)\left(\begin{array}{l}
2 / 3 \\
1 / 3
\end{array}\right) \text { gives }\left(\begin{array}{l}
0.3167 \\
0.3633 \\
0.3200
\end{array}\right)
$$

as the weights assigned to $A, B, C$. He should pay most of his attention to $B$.

The notion of complexity is likely to become very central, and may lead to new characterizations of vagueness both in perception (input) and the specification of action (output). Similarly, the concepts of worrisomeness may best be captured by explicitly using its fuzziness. Th $\curvearrowright$ ideas contributed in this paper constitute a step toward such a clarification and illustrate a useful way to develop Fuzzy Set Theory.

\section{Peferences}

[1] S.H. Barondes ed., Neuronal Recognition (Plenum, New York, 1976).

[2] E. de Bono, New Think: The Use of Lateral Thinking in the Generation of New Ideas (Basic Books, New York, 1968). 
[3] G.J. Chaitin, Algorithmic information theory, IBM J. Res. Develop. 21 (1977) 350-359.

[4] L. Dubins, Group decision devices, Am. Math. Monthly 84(5) (1977) 350-356.

[5] M. Eigen, Molecules, information, and memory: From molecular to neural networks, in: F.O. Schmitt and F.G. Worden, eds., The Neurosciences: Third Study Program (M.I.T. Press, Cambricige, MA, 1974) xix-xxvii.

[6] E.V. Evarts, Sensorimotor cortex activity associated with movements triggered by visual as compared to somesthetic inputs, in: F.O. Schmitt and F.G. Worden, eds., The Neurosciences: Third Study Program (M.l.T. Press, Cambridge, MA, 1974) 327-337.

[7] B.R. Gaines, Foundations of fuzzy reasoning, Internat. J. Man-Machine Studies 8 (1976) 623-668.

[8] B.R. Gaines and L.F. Kohost, The fuzzy decade: a bibliography of fuzzy systems and closely related topics, Int. J. Man-Machine Studies 9 (1977) 1-68.

[9] P. Glansdorff and I. Prigogine, Thermodynamic Theory of Structure, Stability and Fluctuations (Wiley, New York, 1971).

[10] C.H. Graham, Visual perception, In: S.S. Stevens, ed., Handbook of Experimental Psychology (Wiley, New York, 1951) 868-9:

[11] G. Gurin, J. Veroff and S. Feld, Americans View Their Mental Health (Basic Books, New York, 1960).

[12] J. Hadamard, The Psychology of Invention in the Mathematical Field (Princeton University Press Princeton, NJ, 1945).

[13] L.D. Harmon and B. Julesz, Masking in visual recognition: Effects of two-dimensional filtered noise, Science 180(4091) (1973) 1194-1197.

[14] I.L. Janis and L. Mann, Copine, with decisional conflict, Am. Sci. 64(6) (1976) 657-667.

[15] A.R. Kessler and I.D. Pool, Crisiscom: A computer simulation of human information processing during a crisis, IEEE Trans. Systems, Man, and Cybernet. SSC-1(1) (1965) 52-58, 5B.

[16] D.E. Knuth, Mathematics and computer science: Coping with finiteness, Science 194(4271) (1976)1225-1242.

[17] M. Kochen, Control of attention in raising consciousness about community issues, Proc. Second Int. Research Forum on Information Science (SIRE), Copenhagen, Denmark (1978).

[18] M. Kochen and K.W. Deutsch, Decentralization: Toward a rational theory (Unpublished manuscript, MHRI, University of Michigan, Ann Arbor, MI, 1977) (Submitted to Harvard University Press).

[19] L.S. Kubie, Neurotic Distortion of the Creative Process (Noonday Press/Farrar, Straus and Giroux, New York, 1958).

[20] G.A. Miller and N. Chomsky, Finitary models of language users, in: R.D. Luce, ed., Handbook of Mathematical Psychology, Vol. II (Wiley, New York, 1963) 419-491.

[21] G.A. Miller, E. Galanter and K. Pribram, Plans and the Structure of Behavior (Holt, Rinehart and Winston, New York, 1960).

[22] M.A. Morrison and J. Brillhart, A method of factoring and the factorization of F7, Math. Comp. 29 (1975) 183-205.

[23] G. Oster, A. Perelson and A. Katchalsky, Network thermodynamics: Analysis of biophysical systems, Quart. Rev. Biophys. 6 (1973) 1-138.

[24] H. Poincare, Science and Method (Dover Publications, New York, originally published 1914).

[25] G. Polya, Patterns of Plausible Inference (Princeton University Press, Princeton, NJ, 1969).

[26] E.A. Rubinstein and G.V. Coelho, eds., Behavioral Sciences and Mental Health: An Anthology of Program Reports (PHS Publ. No. 2064, NIMH, U.S. Dept. of HEW, Chevy Chase, MD, undated).

[27] T.L. Saaty, Exploring the interface between hierarchies, multiple objectives and fuzzy sets, Fuzzy Sets and Systems 1 (1978) 57-68.

[28] L.A. Zadeh, Fuzzy sets, Information and Controi 8 (1965) 338-353.

[29] F. Zwicker, G. Flottorp and S.S. Stevens, Critical band width in loudness summation, J. Acoust. Soc. Am. 29 (1957) 548-557. 